\title{
BPI Percentage Relief From Pain Treatment or Medication Likert Scale
}

National Cancer Institute

\section{Source}

National Cancer Institute. BPI Percentage Relief From Pain Treatment or Medication

Likert Scale. NCI Thesaurus. Code C120130.

A scale for the subjective scoring of the percentage of relief from pain medication or treatment that ranges from 0\%: No Relief to 10: Complete Relief. 